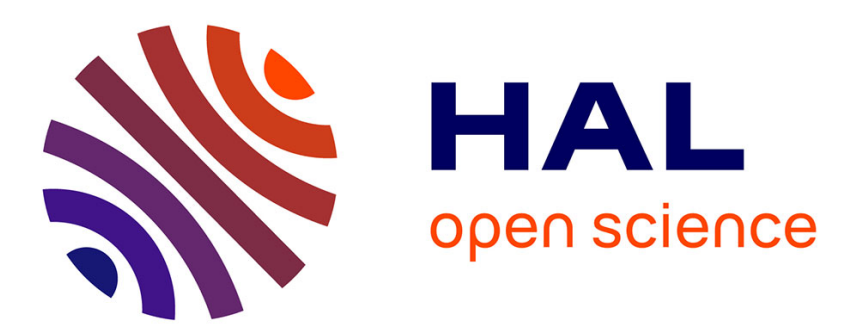

\title{
Impact of Solvent Structuring in Water/ tert -Butanol Mixtures on the Assembly of Silica Nanoparticles to Aerogels
}

Robert Winkler, Elisa Ré, Guilhem Arrachart, S Pellet-Rostaing

\section{- To cite this version:}

Robert Winkler, Elisa Ré, Guilhem Arrachart, S Pellet-Rostaing. Impact of Solvent Structuring in Water/ tert -Butanol Mixtures on the Assembly of Silica Nanoparticles to Aerogels. Langmuir, 2019, 35 (24), pp.7905-7915. 10.1021/acs.langmuir.9b00655 . hal-02161599

\section{HAL Id: hal-02161599 \\ https://hal.umontpellier.fr/hal-02161599}

Submitted on 25 Oct 2021

HAL is a multi-disciplinary open access archive for the deposit and dissemination of scientific research documents, whether they are published or not. The documents may come from teaching and research institutions in France or abroad, or from public or private research centers.
L'archive ouverte pluridisciplinaire HAL, est destinée au dépôt et à la diffusion de documents scientifiques de niveau recherche, publiés ou non, émanant des établissements d'enseignement et de recherche français ou étrangers, des laboratoires publics ou privés. 


\section{Impact of the solvent structuring in water/tert- butanol mixtures on the assembly of silica nanoparticles to aerogels}

Robert Winkler*, Elisa Ré, Guilhem Arrachart* and Stéphane Pellet-Rostaing

ICSM, CEA, CNRS, ENSCM, Univ Montpellier, Marcoule, France

*Corresponding authors ( robert.winkler@cea.fr / guilhem.arrachart@,cea.fr )

\section{Keywords}

nanoparticles, aerogel, sol-gel process, tert-butanol/water, structured solvent, surfactant-less microemulsion 


\begin{abstract}
Soft matter structure is a useful tool for the preparation of well-structured inorganic materials. Here, we report a strategy using a structured solvent based on binary mixtures as directing agent for silica nanoparticles in aerogel elaboration. Binary mixtures involving water/EtOH and water/tert-butanol have been respectively chosen as representatives of unstructured and structured solvents. The systems water/alcohol/TEOS were effectively characterized as surfactant free microemulsions. The enhanced solvent structuring, however, disappears upon the reaction of TEOS and assembly is directed by solvent structuring found in the binary. For the first time, the influence of solvent composition on the sol-gel reaction was investigated with respect to the reaction rate and the structuring behavior thanks to dynamic light scattering (DLS), small and wide angle X-ray scattering (SWAXS) and transmission electron microscopy (TEM) experiments. The silica nanoparticles aggregate in a different manner depending on the solvent composition, which allow changing the morphology, degree of interconnection and surface area of the resulting material. Silica nanoparticles with very high surface area up to $2000 \mathrm{~m}^{2} / \mathrm{g}$ can be obtained by this approach.
\end{abstract}




\section{Introduction}

Silica aerogels are industrially widely applied materials ${ }^{1-4}$ due to their unique properties such as low thermal conductivity, low refractive index optical transmission, low density and high specific surface $\operatorname{areas}^{5}$. Especially their high porosity and high specific surface area make them a useful choice for many applications like thermal insulation ${ }^{6}$, oil-water separation ${ }^{7}$, analytics ${ }^{8}$ and depollution 9 . Furthermore, their production is nowadays easily scalable. The crucial factor of aerogel synthesis is the conservation of the wet gel structure during drying. To this end, the capillary forces must be minimized by using a low surface tension solvent during drying like supercritical $\mathrm{CO}_{2}{ }^{10}$ To create wet gels that exhibit the desired properties, well-reported templating approaches can be used. ${ }^{11-15}$ Also, high internal phase emulsions can lead to foams exhibiting interesting properties. ${ }^{16}$ Conventionally, large, self-assembling molecules known as surfactants are used because their free energy of structure formation is strongly favorable. ${ }^{17}$ However, in a recent study ${ }^{18}$, the effect of water/ethanol/dichloromethane microemulsion on the ammonia catalyzed silica polymerization was investigated. They found a connection between the type of microemulsion $(\mathrm{W} / \mathrm{O} \text {, bicontinuous or } \mathrm{O} / \mathrm{W})^{19}$ and the obtained silica morphology, thus, proving the possibility of templating by surfactant-less microemulsions. In this paper, we investigate the fundamental aspects of using structured solvents in silica aerogel elaboration.

Such structures have been observed in mixtures of small amphiphilic molecules like ethanol $(\mathrm{EtOH})$ or -most prominently- tert-butanol (TBA) and a hydrophilic solvent. ${ }^{20-25}$ The addition of hydrophobic molecules like 1-octanol amplifies this structuring. ${ }^{26-29}$ Here, subtle balances between hydration forces, entropy and curvature play important roles in the solvent structuring on the mesoscale. ${ }^{30-32}$ It is more reasonable to speak of fluctuating hydrophobic and hydrophilic domains instead of defined structures. In spite of their shapeless nature, such compartments show an influence on chemical reactivity. ${ }^{33-36}$ 
Among the studies on chemical reactions in such structured solvents, very few deal with the synthesis of polymers ${ }^{37}$ or the sol-gel process ${ }^{18}$. Another recent study ${ }^{38}$ showed that even the presence of binary mixtures of a deep-eutectic solvent and water is able to influence the silica morphology. A surprising result was reported by Wang et a. $1^{39}$ where a small amount of a cosolvent is responsible for the formation of silica nanosheets.

In this study, the sol-gel process is performed in a system that contains only the essential components: tetraethyl orthosilicate (TEOS) as Si-source, water for hydrolysis, an alcohol to assure solubilization and $\mathrm{HCl}$ as catalyst. TEOS content and $\mathrm{HCl} / \mathrm{TEOS}$ ratio are kept constant, in order to reduce experimental parameters. To investigate the effect of structuring, like mentioned before, TBA is the ideal candidate. ${ }^{23}$. For comparison, EtOH was chosen as nonstructuring counterpart. 


\section{Results and discussion}

\section{Phase behavior of the ternary systems}

In order to determine the solubility of TEOS in the binary mixtures of alcohol/water and to choose the most suitable sol compositions, first, the phase diagrams of the systems TEOS/alcohol/water were determined. Since TEOS hydrolyzes very fast at low $\mathrm{pH}$, the phase diagram was prepared without catalyst addition. The phase diagrams are presented in Figure 1. Sample compositions are also displayed. "A" to "G" denote both $A_{C 2}$ and $A_{C 4}$ to $G_{C 2}$ and $G_{C 4}$. For presentability in Figure 1, the water and the $\mathrm{HCl}$ weight fraction in the prepared samples (see Table 2 for compositions) were added to give " $w_{\text {water" }}$ in the diagram.

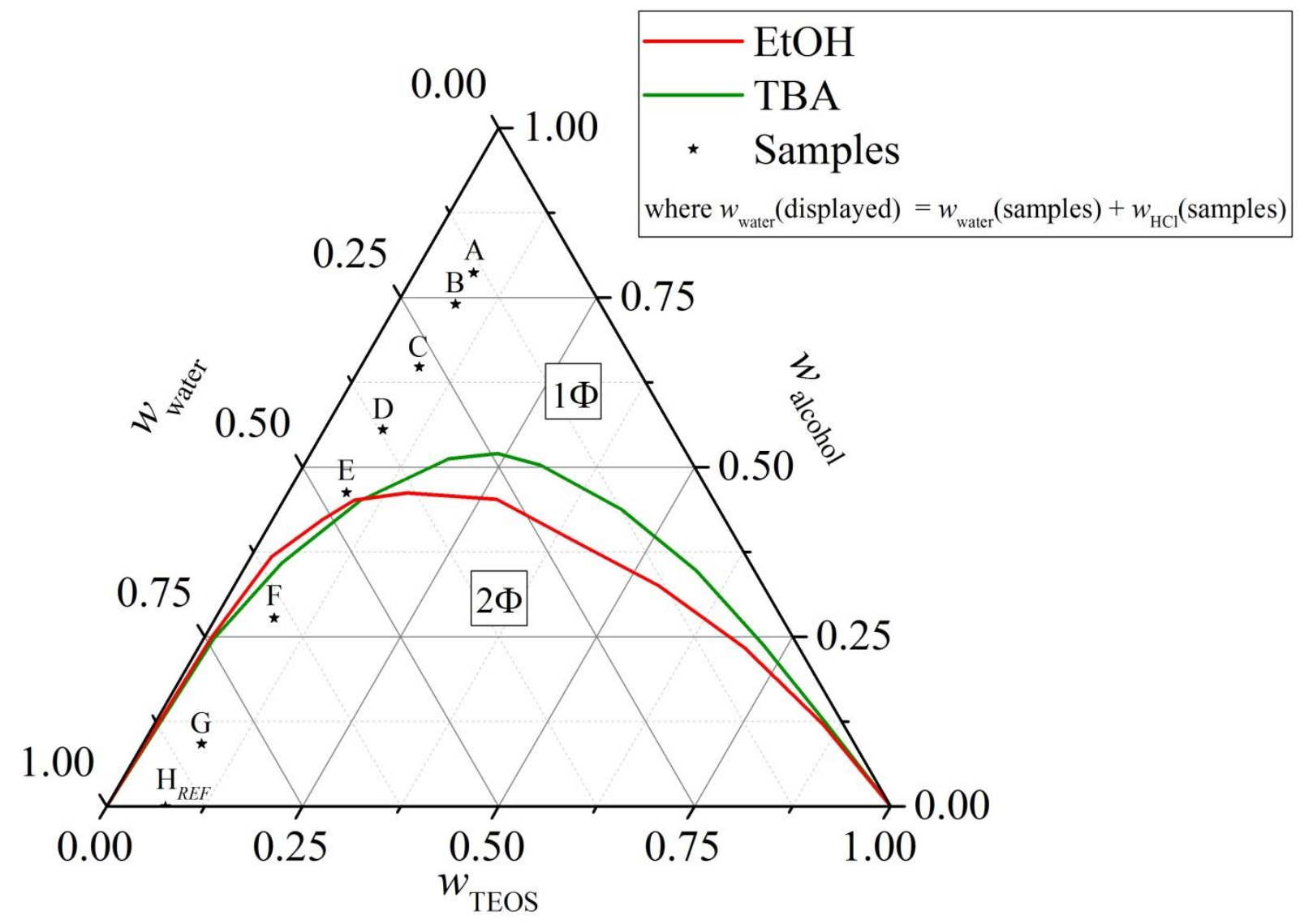

Figure 1. Ternary phase diagrams of the systems water/TBA/TEOS (-) and water/EtOH/TEOS (-) in weight fractions determined at $25 \pm 0.5^{\circ} \mathrm{C}$. The mono- and biphasic regions are indicated in the respective areas. The selected sols are referred A to H (see Table 2 for compositions). "A" to "G" denote both $A_{C 2}$ and $A_{C 4}$ to $G_{C 2}$ and $G_{C 4}$. For presentability, the water and the $\mathrm{HCl}$ 
weight fraction in the prepared samples (see Table 2 for compositions) were added to give " $w_{\text {water" }}$ " in the diagram.

Both phase diagrams show the classical behavior of systems containing two immiscible components, in this case water and TEOS, and a co-solvent, which is completely miscible with the other two. When adding a certain quantity of co-solvent (here: EtOH or TBA) the system can be mixed. The necessary amount to reach the monophasic region depends on the ratio of the immiscible components and the nature of the co-solvent. In addition, for considerations on the solubilization capacity of the co-solvent, a ternary phase diagram in molar fractions is illustrated in the Figure S1 in the Supporting Information. The biphasic area is slightly smaller with TBA than with EtOH. Several reasons for this difference are identified: (i) the nature of the co-solvent, i.e. its polarity or its molecular structure ${ }^{17,30,40}$, and (ii) the co-solvent-co-solvent and co-solvent-solute interactions. ${ }^{29,41}$ According to earlier work ${ }^{29}$, the high concentration of a solute with weak co-solvent-solute interactions favors solubilization by the integration of the solute in a hydrophobic pseudophase. In our case, the presence of a sufficient amount of TBA leads to a pre-structuring where the hydrophobic TEOS can be solubilized. In the case of EtOH, this pre-structuring does not exist and the solubilization of strongly hydrophobic compounds is less efficient.

\section{Impact of the TEOS addition to the sol structuring}

To gain further information on the samples, their structuring on the mesoscale was investigated by SWAXS. The scattering patterns of the samples without $\mathrm{HCl}$ just after the addition of the 7.5 w\% TEOS and of their associated binary mixtures are shown in Figure 2. The data in Figure 2 c) were already published earlier. ${ }^{29}$ The SAXS spectra of samples Fcx to $\mathrm{H}_{R E F}$ are not displayed in Figure 2 because they are biphasic and, therefore, not comparable to the systems without TEOS. The weight fractions of water of samples $A_{C X}$ were adjusted to 
0.1 in binary mixtures and 0.0925 in ternary mixtures to be compared with the data from the literature.
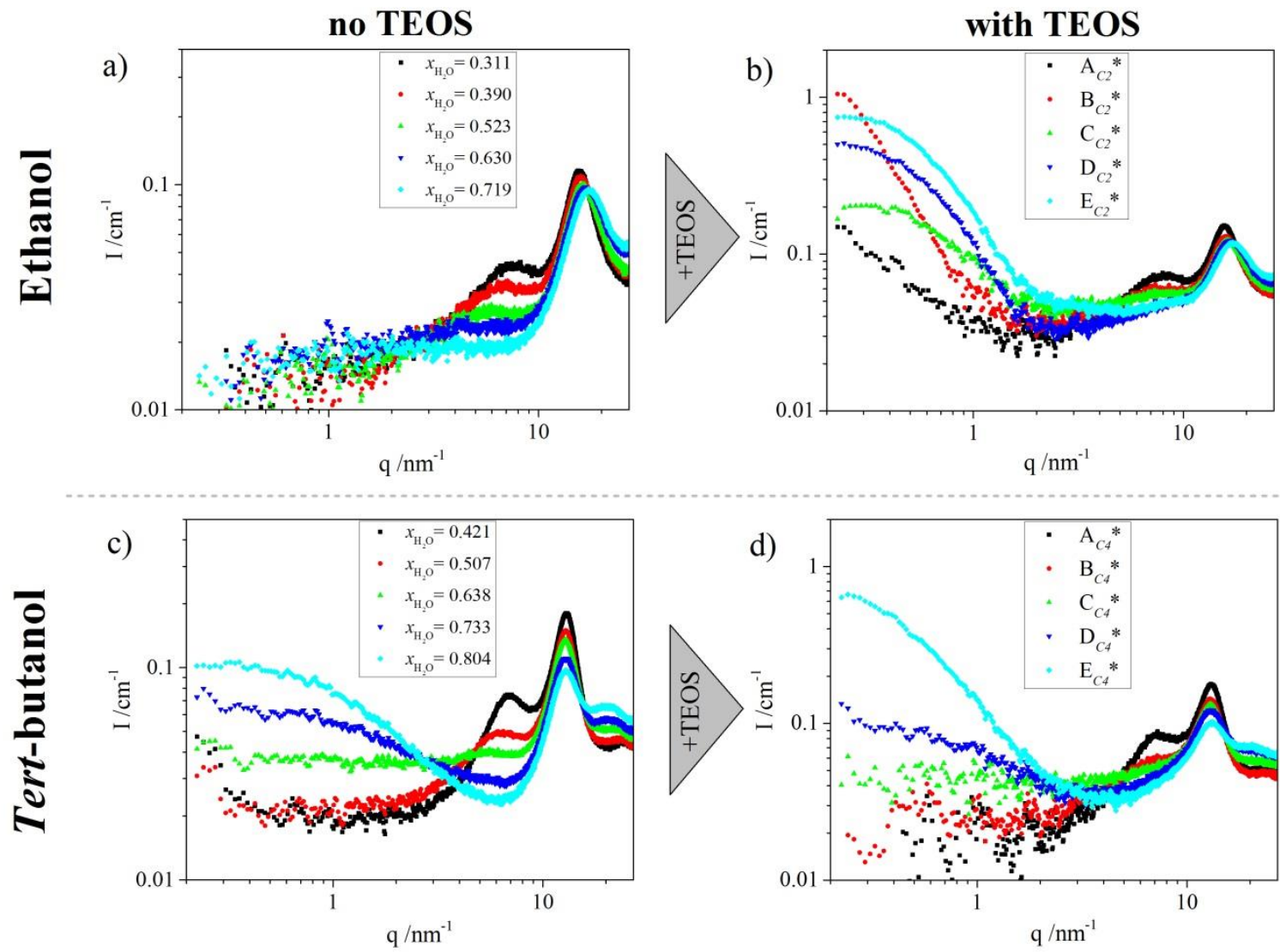

Figure 2. a) and c) SWAXS patterns of binary alcohol/water mixtures. b) and d) SWAXS spectra of ternary TEOS/alcohol/water mixtures at neutral $\mathrm{pH}$ just after the $7.5 \mathrm{w} \%$ TEOS addition. Data for c) was already published earlier. ${ }^{29}$ Sample compositions of ternary mixtures are given in Table 3.

These SWAXS patterns display several results which depend on the solvent. First, at high $q$ $\left(q>3 \mathrm{~nm}^{-1}\right)$ several peaks attributed to the various pair correlations are visible. First, the peak at $20 \mathrm{~nm}^{-1}$ assigned to $\mathrm{O}-\mathrm{O}$ pair correlations in the $\mathrm{H}$-bonding network of water are visible in all patterns. It shifts slightly to smaller $q$ with increasing alcohol contents. This corresponds to an increase of the distances in real space and may indicate the partial disruption of the H-bonded network. ${ }^{42}$ Second, the peaks at $15.5 \mathrm{~nm}^{-1}$ for the samples with EtOH and at $13 \mathrm{~nm}^{-1}$ for the samples with TBA corresponds to characteristics distances of approximately $0.4 \mathrm{~nm}$ and $0.5 \mathrm{~nm}$ 
respectively and are assigned to the pair correlations between the alkyl chains. ${ }^{43}$ Whereas their intensity depends on the alcohol content, their position does not shift significantly with the concentrations. The apparent shift of this peak to higher $q$ in EtOH containing samples is due to the superposition with the peak assigned to O-O pair correlations. The apparent broadening upon addition of TEOS is due to the apparition of Si-Si pair correlations at $9 \mathrm{~nm}^{-1}$ and $16 \mathrm{~nm}^{-1}$. In these mixtures, the peaks of TEOS are not clearly visible due to its low concentration. Thus, the positions of these peaks were determined using the SWAXS spectrum of pure TEOS. (see Figure S2 in the Supporting Information). Third, the peaks at $8 \mathrm{~nm}^{-1}(0.8 \mathrm{~nm}$ in real space) for EtOH and at $7 \mathrm{~nm}^{-1}(0.9 \mathrm{~nm}$ in real space) for TBA are attributed to the O-O correlation distance of the alcohol $-\mathrm{OH}$ groups. ${ }^{43}$ Accordingly, the peak intensity decreases with lower alcohol contents.

In the low $q$ region of the pattern $\left(q<3 \mathrm{~nm}^{-1}\right)$, significant differences between the two cosolvents can be noticed. In the samples with $\mathrm{EtOH}$, the addition of TEOS, leads to a significant scattering increase presenting various slopes depending on the mixture: For the samples $\mathrm{A}_{C 2}$ and $\mathrm{B}_{C 2}$ a constant increase $\left(q^{-1}\right.$ for $\mathrm{A}_{C 2}$ to $q^{-2.4}$ for $\mathrm{B}_{C 2}$ ) and no plateau are visible. For sample $\mathrm{C}_{C 2}, \mathrm{D}_{C 2}$ and $\mathrm{E}_{C 2}$ a plateau is reached. The plateau shifts to lower $q$ values with increasing water contents. In the samples with TBA without TEOS, the scattering intensity increases with the water content from $x_{\text {water }}>0.638$. A plateau is visible that shifts to lower $q$ with increasing water content. After the TEOS addition, this scattering intensity increases noticeably whereas the shape remains the same (around one order of magnitude for sample $E_{C 4}$ ) and the plateau shifts to lower $\mathrm{q}$.

This intensity increase at low $q$ indicates a structuring at the mesoscale (According to IUPAC 2 to $50 \mathrm{~nm}) .{ }^{44}$ In the case of EtOH two different behaviors are observed. For lower water content $\left(\mathrm{A}_{C 2}\right.$ and $\mathrm{B}_{C 2}$ ), the maximum intensity is not inside the observation window indicating organization on a very large scale. The higher slope for $\mathrm{B}_{C 2}$ compared to $\mathrm{A}_{C 2}$ indicates a 
structuring change. For $\mathrm{C}_{C 2}$ and the samples with higher water contents, the intensity limit is in the observed $q$-range. This may be explained by the formation of large structures with trace amounts of highly hydrophobic compounds. ${ }^{28}$ In this case, the highly hydrophobic compounds may be the polycondensed oligomers of TEOS which are formed in the commercial product after the first opening due to air humidity. ${ }^{45}$ Thus, the excess scattering at low-q for samples $\mathrm{A}_{C 2}$ and $\mathrm{B}_{C 2}$ is assumed to be caused by large shapeless aggregates. When adding more water, these aggregates get increasingly constrained by the H-bonding network of water formed around them $\left(\mathrm{B}_{C 2}\right)$ until reaching objects of less than $30 \mathrm{~nm}$ in size $\left(\mathrm{C}_{\mathrm{C} 2}\right.$ to $\left.\mathrm{E}_{\mathrm{C} 2}\right)$. In the binary system TBA/water, organization is due to rapidly fluctuating water- or TBA rich aggregates. ${ }^{29}$ This is typical for short chain alcohol/water mixtures..$^{20-24}$ The visibility of these aggregates on SAXS patterns depends on their size and on their electronic density contrast to the rest of the solution. In EtOH/water mixtures, for example, the aggregates are too small and do not have a sufficiently high contrast to their surroundings to be visible. ${ }^{22}$ When adding the hydrophobic TEOS a transition to a "pre-Ouzo" system is observed. Depending on the type and the weight percentage of co-solvent this leads to an enhanced structuring. ${ }^{29,30,46}$ In the case of TBA, the preformed structures are swollen by TEOS and this is visible on SAXS pattern by an increase of the intensity and the plateau shift to smaller $q$. This is due to a higher solubility of TEOS in the TBA-rich domains than in the water-rich domains Samples which do not present any scattering intensity at low $q$ in the binary mixtures remain unchanged upon addition of TEOS. A size analysis of the aggregates in the structured systems gives insight on its morphology. Characteristic sizes were evaluated by the fitting of SAXS data. Classically, the SAXS patterns of surfactant-free microemulsions can be fitted using then Ornstein-Zernicke (OZ) function. The samples $\mathrm{D}_{C 4}$ and $\mathrm{E}_{C 4}$ and their corresponding binary mixtures show good agreement with the fit. In the samples without TEOS correlation lengths of $0.65 \mathrm{~nm}$ for the binary mixture corresponding to $\mathrm{D}_{C 4}$ and $0.77 \mathrm{~nm}$ for the binary mixture corresponding to $\mathrm{E}_{C 4}$ are obtained. 
This is in agreement with literature. ${ }^{29}$ In the samples with TEOS, correlation lengths of $0.88 \mathrm{~nm}$ for $\mathrm{D}_{C 4}$ and $2.82 \mathrm{~nm}$ for $\mathrm{E}_{C 4}$ have been found. Supposing spherical aggregates, the respective aggregate dimensions are $3.52 \mathrm{~nm}$ and $11.23 \mathrm{~nm}$. The samples $\mathrm{C}_{C 2}, \mathrm{D}_{C 2}$ and $\mathrm{E}_{C 2}$ are fitted increasingly worse using the OZ function because the slopes at low- $q$ are too steep. However, approximatively, correlation lengths between $1.8 \mathrm{~nm}\left(\mathrm{C}_{C 2}\right)$ and $2.5\left(\mathrm{E}_{C 2}\right)$ are obtained. The results are in agreement with an $\mathrm{O} / \mathrm{W}$ microemulsion. The addition of TEOS enhances structuring by (I) swelling the existing structure in the case of TBA or (II) inciting the aggregation of the co-solvent around it in the case of EtOH. When decreasing the alcohol/water ratio (i.e. DC4 $\rightarrow \mathrm{EC} 4$ ), the system will minimize the interphase formed by the alcohol. This is done by increasing the aggregate size. The results agree with the literature for similar systems. A swollen network of H-bonded groups is probably at the origin of the structuring. ${ }^{47}$ For ternary mixtures with low hydrophobic phase content close to the demixing line, structures resembling $\mathrm{O} / \mathrm{W}$ aggregates were proposed. ${ }^{48}$

To summarize, ternary mixture TEOS/alcohol/water (where "alcohol" can be EtOH or TBA) are typical surfactant-free microemulsions. The components take their roles as hydrophilic component (water), hydrophobic component (TEOS) and co-solvent or -more generally hydrotrope (EtOH or TBA). The organization of components at the mesoscale increases when approaching the phase boundary. ${ }^{27,49,50}$ The type and the extend of structuring depends on the co-solvent. When using TBA, the aggregation is heavily influenced by structuring which is already present in the binary mixture TBA/water. They will allow the TEOS solubilization. With EtOH, structures which allow solubilization of TEOS are formed only in the ternary system. $^{27,29}$ 


\section{Study of the sol-gel transition}

After the addition of $\mathrm{HCl}$ used as a catalyzer to initiate the TEOS hydrolysis, the durations before gelification at $50^{\circ} \mathrm{C}$ were determined by naked eye for both series. To confirm the results obtained by these observations for the TBA series and to reduce the error margins, gel time determination was also performed by DLS. Upon TEOS addition, a slight heating was noticed that indicates the start of hydrolysis. The results are presented in Figure 3.

a)

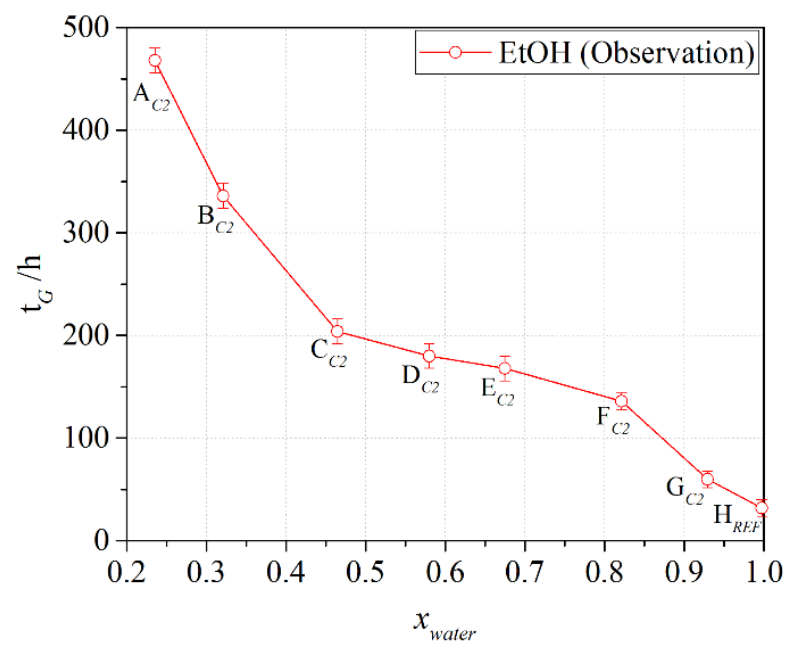

b)

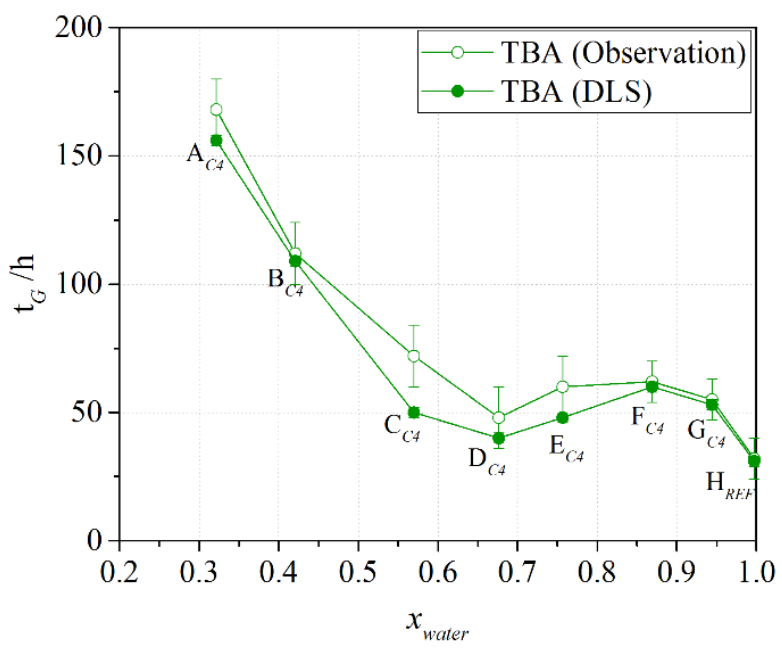

Figure 3. Gel times of the samples as a function of the molar fraction of water $\mathrm{x}_{\text {water }}$ as determined by observation and by DLS.

Comparing the results obtained by observation and DLS, overall shorter times were observed with the DLS method. However, all trends remain consistent. First, the gel times of the sols with $\mathrm{EtOH}$ at $x_{\text {water }}<0.7$ are three times longer than the ones with TBA. This difference is reduced when the water content increases. Second, whatever the solvent, the gel time globally decreases with increasing water content. This evolution follows three stages. Up to $x_{\text {water }}=0.5$, the gel time decreases almost linearly with the water content. For $x_{\text {water }}$ higher than 0.5 , the gel time decrease follows two different behaviors depending on the solvent. For sols containing $\mathrm{EtOH}$, the gel time decreases linearly from $x_{\text {water }}=0.5$ to $x_{\text {water }}=0.8$ with a lower slope than at lower water contents. For sols containing TBA, the gel time evolution between $x_{\text {water }}=0.5$ and 
$x_{\text {water }}=0.8$ presents a minimum for the sample $\mathrm{D}_{C 4}\left(x_{\text {water }}=0.677\right)$. Finally, at high water contents $\left(x_{\text {water }}>0.9\right.$ for TBA and $x_{\text {water }}>0.8$ for EtOH), a strong decrease of gel time is observed. Gel-time depends on the volume fraction occupied by the growing aggregate. Consequently, the aggregate structure has an important influence.

To investigate the morphology of the growing structures with the gel time, the structure evolution was followed by SAXS. The SAXS pattern evolution of the two sols $\mathrm{C}_{C 4}$ and $\mathrm{C}_{C 2}$ were chosen to illustrate the behavior and are presented in Figure 4 a).
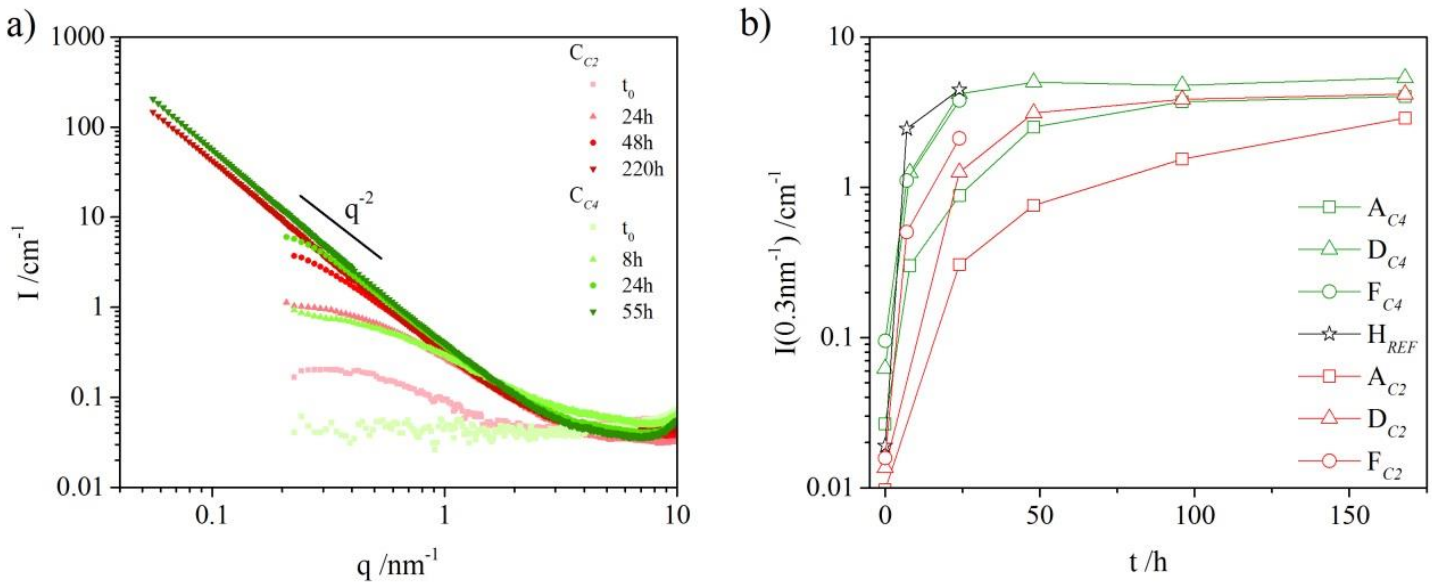

Figure 4. a) Evolution of the SAXS patterns of the sols $\mathrm{C}_{\mathrm{C} 4}$ and $\mathrm{C}_{\mathrm{C} 2}$ during the sol-gel transition. The symbol color intensifies with sample ageing. b) evolutions of the scattering intensity at $\mathrm{q}=0.3 \mathrm{~nm}^{-1}$ of the SAXS patterns as a function of time.

As discussed earlier, immediately after TEOS addition, the observed scattering originates from the mesoscale organization of unreacted components ( $t_{0}$ in Figure 4 a)). During the polymerization, the low $q$ scattering develops the same way for all sols. Three aspects should be highlighted. First, all SAXS patterns present a slope proportional to $\sim q^{-2.1}$ (Figure 4 a)). A plateau marks the end of this slope at low $q$. The evolution rate of this plateau is proportional to the gel time as highlighted by the scattering patterns of $\mathrm{C}_{C 4} 8 \mathrm{~h}$ and $\mathrm{C}_{C 2} 24 \mathrm{~h}$ recorded at similar points during the polymerization $\left(\frac{t}{t_{G}} \approx 0.12\right)$. During polymerization the plateau shifts to 
increasingly low $q$ and higher intensities. Finally, after $\frac{t}{t_{G}} \approx 0.3$ no plateau is observable. Second, the slope is proportional to $\sim q^{-2.1}$ giving evidence of the nature of polymerization. Third, the initial organization of the water and the alcohol is not visible anymore after TEOS addition probably because its scattering is hidden under that of the developing silica polymer.

The evolution of the low-q scattering pattern is characteristic for objects that primarily increase in size and not in number. ${ }^{10}$ Logically, the disappearance of the plateau after $\frac{t}{t_{G}} \approx$ 0.3 indicates that the correlation lengths become too large for the observation window. In Figure $4 \mathrm{~b}$ ), the scattering intensities of selected samples at $q=0.3 \mathrm{~nm}^{-1}$ as a function of time are plotted. For all samples, like mentioned before, an increasing intensity is observed at longer times. The intensity increase is accelerated at higher water contents. The acceleration is directly correlated to the gel time of the samples. The shorter the gel times the faster the intensity increases. This is also observed for the development of Si-O-Si bonds as observed in the FTIR spectra (see Figure S3 in the Supporting Information).

The constant slope of $\sim q^{-2.1}$ is characteristic of the formation of fractal objects via a reaction limited cluster-cluster growth (RLCA) or a diffusion limited cluster-cluster growth (DLCA). ${ }^{10,51,52}$ Evidence has shown that rearrangements can lead to the same fractal dimensions. ${ }^{53}$ From the SAXS spectra we conclude that the polymerization rate is proportional to the gel time observed for the sols. Polymerization occurs via cluster-cluster aggregation of primary particles that are formed early in the process. The structural evolution is identical for all samples.

With the obtained structural information, the two different behaviors with EtOH and TBA in the region of samples $A_{C X}$ to $F_{C X}$ can be explained by several experimental factors. In order to simplify the explanation, it is assumed that hydrolysis was very advanced in all samples after several minutes and, thus, does not contribute to the gel time. This last assumption is reported in literature ${ }^{54,55}$ and supported by the fact that under acidic conditions, the hydrolysis is 
generally faster than condensation and the high $\left[\mathrm{H}_{2} \mathrm{O}\right] /[$ TEOS $]$ ratio $\left(\left[\mathrm{H}_{2} \mathrm{O}\right] /[\right.$ TEOS $]=15.7$ for samples $A_{C X}$ ) increases that effect. ${ }^{10}$ This is also visible in the FTIR spectra of all samples which display a peak at $950 \mathrm{~cm}^{-1}$ corresponding to the $\equiv \mathrm{Si}-\mathrm{O}$ stretching of non-hydrolyzed TEOS only just after the TEOS addition. Moreover, the initially biphasic sample $\mathrm{H}_{R E F}$ becomes monophasic after 3 min of vortexing due to the increasing hydrophilicity of hydrolyzed species and the production of solubilizing EtOH during the reaction.

The first factor which affects the condensation rate is the concentration of catalytic species. A higher concentration increases the hydrolysis/condensation rate. Here, the catalysis occurs via the protonation of silica species and is proportional to the $\mathrm{H}_{3} \mathrm{O}^{+}$concentration. ${ }^{52}$ The qualitative inspection of FTIR spectra (see Figure S4 in the Supporting Information) recorded along the polymerization showed that, indeed, the concentration of $\mathrm{H}_{3} \mathrm{O}^{+}$increases with increasing water content. This is accordance with the observed trends.

Second, the re-esterification of hydrolyzed species may also impact the gel time. Indeed, partial re-esterification (RE) of hydrolyzed species is possible due to the reversibility of the hydrolysis reaction and can occur with reactive alkoxy species. RE is also favored under acidic conditions. ${ }^{10}$ TBA is too unreactive whereas EtOH is sufficiently reactive for this process to be considered. ${ }^{56,57}$ For our two systems, due to the high concentrations of EtOH compared to the strong dilution of silicate species, it is probable that RE leads to an overall decrease of silicate species reactivity. This effect is more pronounced at higher alcohol concentrations. In mixtures with high water content $\mathrm{x}_{\text {water }}>0.8$ this effect vanishes.

Third, the reaction rate is influenced by the repulsive forces between the charged primary particles and their diffusion. This is described by the aggregation rate constant $k$, as described in the Supporting Information. The values were calculated from the primary particle radii from the SAXS measurements (Table 1) and the dielectric constants (see Figure S5 in the Supporting Information) and the viscosities (see Figure S6 in the Supporting Information) from literature. 
Overall, the estimated reaction rate increases at increasing water concentration (see Figure S7 in the Supporting Information). For one, the viscosity decreases at increasing water concentration. Thus, the diffusion increases and objects potentially encounter each other more frequently. The encounters are moderated by repulsive forces between the objects. The increasing dielectric constant at increasing water concentrations attenuates the repulsive forces and the reaction rate accelerates.

While most of the gel time tendencies can be explained by these factors, the minimum of gel time for the sample $\mathrm{D}_{C 4}$ cannot be explained by any of the mentioned factors because they describe continuous trends. A hypothesis allowing the explanation of this behavior will be proposed in the discussion part. 


\section{Influence of the sol composition on material structure}

In order to obtain aerogels, first, a solvent exchange with EtOH was performed to induce the re-esterification of the materials surface making it more hydrophobic. ${ }^{58,59}$ This re-esterification reduces the capillary forces during solvent removal and, thus, minimizes the material modification during drying. Second, another solvent exchange with acetone and $\mathrm{SC} \mathrm{CO}_{2}$ was performed. Acetone was used for its high solubility in $\mathrm{SC} \mathrm{CO}_{2}$. The obtained materials were analyzed by nitrogen adsorption-desorption experiments. The specific surface areas $\mathrm{S}_{B E T}$ obtained as a function of $x_{\text {water }}$ are shown in Figure 5.

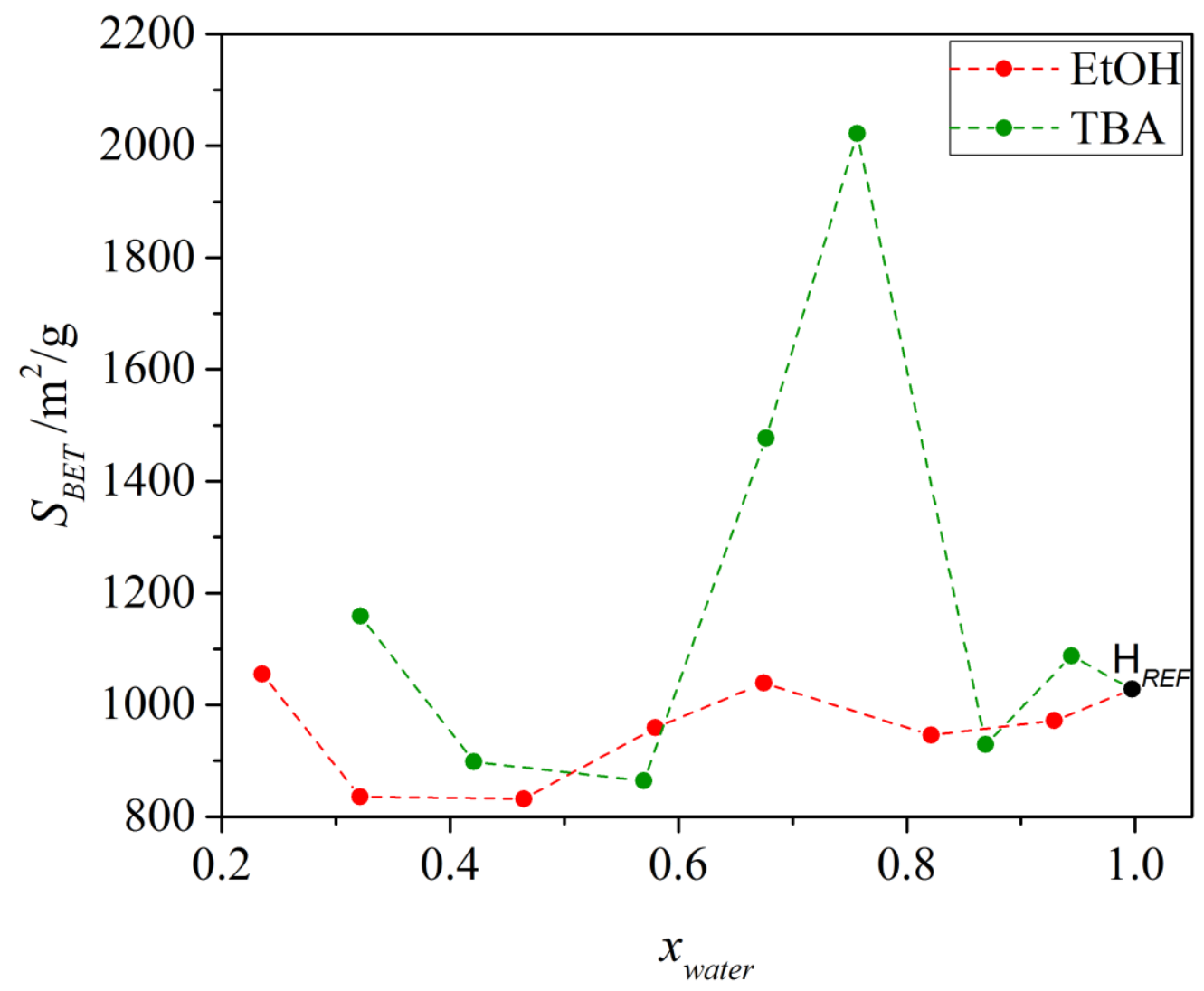

Figure 5. Specific surface areas $\left(\mathrm{S}_{\mathrm{BET}}\right)$ of aerogels obtained by the BET method as a function of the molar fraction of water $\mathrm{x}_{\mathrm{water}}$ in the corresponding sol. 
Except for the samples $\mathrm{D}_{C 4}$ and $\mathrm{E}_{C 4}$, and whatever the solvent, aerogels have a similar $\mathrm{S}_{B E T}$ of as around $1000 \mathrm{~m}^{2} / \mathrm{g}$. For aerogels obtained from sols $\mathrm{D}_{C 4}$ and $\mathrm{E}_{C 4} \mathrm{~S}_{B E T}$ of $1400 \mathrm{~m}^{2} / \mathrm{g}$ and $2100 \mathrm{~m}^{2} / \mathrm{g}$ are obtained respectively. This result can be related to the particular behavior in the sol-gel process already observed. The solvent of the samples $\mathrm{D}_{C 4}$ and $\mathrm{E}_{C 4}$ showed the most pronounced scattering.(see Figure 2 a)) Furthermore, the samples $D_{C 4}$ and $E_{C 4}$ displayed unexpectedly low gel times.(see Figure 3 a))

To investigate the morphology at a scale of 1 to $30 \mathrm{~nm}$ SAXS was performed (see Figure S8 in the Supporting Information). All samples showed the same scattering intensity evolution characteristic for aerogels produced from one-step acid catalysis. ${ }^{60,61}$ The fractal dimension increases from -2.15 (see Figure 4 a)) to values around -2.7 (see Table S1 in the Supporting Information). From this, a typical porosity shrinkage at small size scales can be concluded. ${ }^{10,62,63}$ The radii of the primary particles $\left(r_{0}\right)$ that form the network were determined by fitting the SAXS patterns (see SI for complete results). The results are shown in Table 1. 
Table 1. The radii (r0) of primary particles as obtained by fitting the SAXS patterns of the aerogels.

\begin{tabular}{|c|c|c|}
\hline Co-solvent & Reference & $\mathrm{r}_{0} / \mathrm{nm}$ \\
\hline \multirow{5}{*}{ Ethanol } & $\mathrm{C}_{C 2}$ & $0.35 \pm 0.04$ \\
\hline & $\mathrm{D}_{C 2}$ & $0.30 \pm 0.04$ \\
\hline & $\mathrm{E}_{C 2}$ & $0.27 \pm 0.03$ \\
\hline & $\mathrm{F}_{C 2}$ & $0.28 \pm 0.03$ \\
\hline & $\mathrm{G}_{C 2}$ & $0.29 \pm 0.03$ \\
\hline \multirow{7}{*}{ Tert-butanol } & $\mathrm{A}_{C 4}$ & $0.23 \pm 0.03$ \\
\hline & $\mathrm{B}_{C 4}$ & $0.32 \pm 0.04$ \\
\hline & $\mathrm{C}_{C 4}$ & $0.32 \pm 0.04$ \\
\hline & $\mathrm{D}_{C 4}$ & $0.36 \pm 0.04$ \\
\hline & $\mathrm{E}_{C 4}$ & $0.28 \pm 0.03$ \\
\hline & $\mathrm{F}_{C 4}$ & $0.26 \pm 0.03$ \\
\hline & $\mathrm{G}_{C 4}$ & $0.23 \pm 0.03$ \\
\hline $\begin{array}{c}\text { Ref. without } \\
\text { alcohol }\end{array}$ & $\mathrm{H}_{R E F}$ & $0.27 \pm 0.03$ \\
\hline
\end{tabular}


The sizes of primary particles can be separated in two domains: In the first, at higher water contents, values between 0.23 and $0.3 \mathrm{~nm}$ are obtained. In EtOH containing samples, this is the case at $x_{\text {water }}>0.58\left(\mathrm{D}_{C 2}\right)$. Whereas in TBA containing samples, this is the case at $x_{\text {water }}>0.757$ $\left(E_{C 4}\right)$. In the second, at lower water contents, the particle size evolution depends on the alcohol. In aerogels produced using EtOH as co-solvent, the radii of primary particles decrease continuously until $\mathrm{D}_{C 2}$. When using TBA as a co-solvent, $\mathrm{r}_{0}$ increases up to a maximum at sample $\mathrm{D}_{C 4}$. The evolution of the primary particle size when using EtOH as a co-solvent can be explained by the experimental factors mentioned above. Since primary particles are formed in the first instants of the reaction, their size is largely controlled by the hydrolysis reaction. At higher alcohol content, the catalytic activity is lowered. Lower catalytic activity leads to slower hydrolysis. When the hydrolysis is slower, the nucleation is slower and the nuclei formed can grow to larger sizes before the TEOS is completely consumed. At $x_{\text {water }}>0.58$, the critical size for the nuclei under these conditions is obtained. When using TBA as co-solvent, the water content where the largest primary particles are found $\left(\mathrm{D}_{C 4}\right)$ can be correlated to the structuring in the binary water/TBA system. ${ }^{29,34}$ Here, an inverse structuring with water-rich domains in a TBA-rich phase was proposed. Since the primary particles grow in the aqueous phase, these water-rich domains can serve as microreactors. Inside, the particle growth is controlled by a lower number of nuclei. Containing the same amount of TEOS, this leads to larger particles. When entering the bicontinious region at higher water contents, a change to nuclei size-limited behavior is observed. 
The samples were also characterized by TEM. (Figure 6) The enlarged images can be found in the SI.
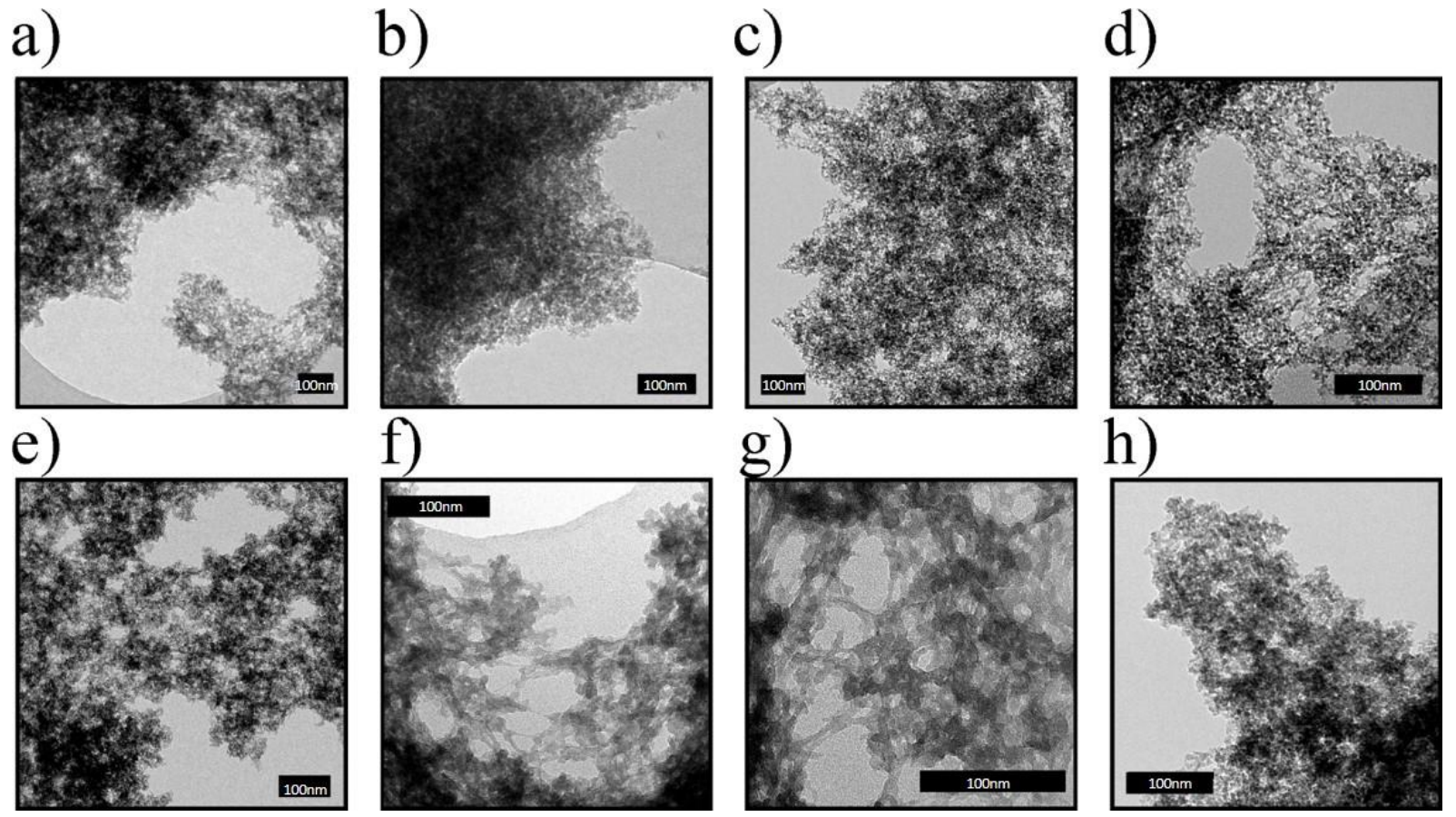

Figure 6. TEM images of aerogels produced from different sols: a) $B_{C 2}$, b) $E_{C 2}$, c) $G_{C 2}$, d) $H_{R E F}$,

e) $A_{C 4}$, f) $\left.D_{C 4}, g\right) E_{C 4}$, h) $F_{C 4}$. Scale bar $=100 \mathrm{~nm}$.

The TEM images show the typical granular structure of aerogels consisting in nanoparticles with radii of 0.2 to $0.8 \mathrm{~nm}$. This agrees well with the sizes obtained by SAXS where radii of 0.23 and 0.36 have been obtained. The structure at the scale of tens of nanometers varies between the samples. Samples where the solvent shows no excess scattering at low $q$ are densely aggregated clusters (see Figure 6 a) - e) and h). Aerogels that were produced in solvents where excess scattering at low $q$ has been observed show an open network at the mesoscale (see Figure $6 \mathrm{f}$ ) and g)). This coincides with the samples that display increased specific surface areas. It is important to distinguish the porosity observed in samples $\mathrm{D}_{C 4}$ and $\mathrm{E}_{C 4}$ from the openings observable in the other samples. In $\mathrm{D}_{C 4}$ and $\mathrm{E}_{C 4}$ the surroundings of pores are like elongated bridges and flat surfaces. The openings observed in the other samples show the roughness at the size scale of primary particles and, therefore, merely represent holes due to 
sample preparation. Thus, TEM images indicate that the particular behavior observed for the sample $\mathrm{D}_{C 4}$ and $\mathrm{E}_{C 4}$ originates from structural differences. 


\section{Discussion}

Binary mixtures of water and alcohol show interesting properties for the application as solvents in the sol-gel process. In the present comparison of $\mathrm{EtOH}$ and $\mathrm{TBA}$, the typical formation of a surfactant-free microemulsion in the ternary mixture of water/alcohol/TEOS was observed. This mesoscale structuring, induced by the addition of hydrophobic TEOS, however, can be considered unimportant for the sol gel process at low $\mathrm{pH}$. The reason is the rapid hydrolysis of TEOS in the examined conditions which leads to the elimination of the hydrophobic compound. Then, the resulting hydrolyzed silicate species react to form sub-nanometer sized primary particles. By further Si-O-Si bond formation between the particles, an open silica network with fractal character is formed. The ratio of water/alcohol or the choice of alcohol does not change the mechanism of polymerization allowing the direct comparison. The network formation proceeds at different rates depending on the water/alcohol ratio which was observed by SAXS, FTIR and gel time measurements. As discussed, several experimental factors are responsible for the different rates. Investigations on the aerogels obtained after drying by $\mathrm{SC} \mathrm{CO}_{2}$, allow the conclusion that, indeed, a network of primary particles was formed during the sol gel process. The aerogels displayed typical properties concerning specific surface areas and SAXS patterns and a typical morphology. However, two samples from the TBA-series display exceptional features that will be discussed in the following. The hypothesis on the mechanism is depicted in Figure 7 in which the water content increases along the axis. The images illustrate the solvent behavior around the silica polymer. Light blue is water-rich phase, dark blue the hydration layer around silica and green the TBA-rich phase. In the picture illustrating pure water and the mixture $25 \mathrm{w} \%$ water in TBA (or TBA in water) the solution is considered as an homogeneous mixture. At the 50/50 mixture the solvent is considered heterogeneous. Here, it creates voids in the silica matrix after the sol-gel process. 


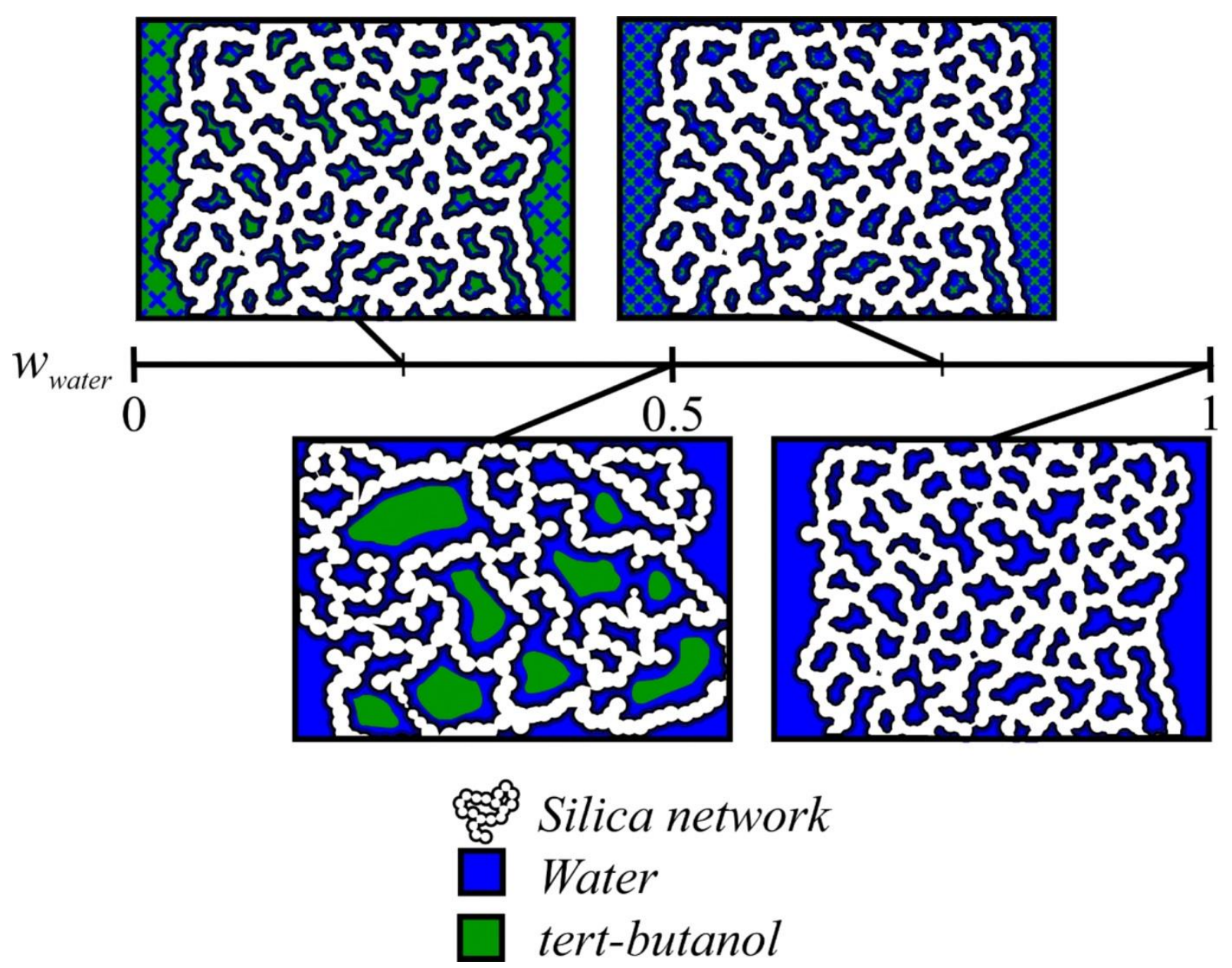

Figure 7. Simplified illustration of the structuring at the mesoscale during the sol-gel process according to the proposed mechanism.

Specific case of the $C_{C 4}$ and $D_{C 4}$ samples: impact of the solvent structuring All the results obtained for the samples, n(TBA)/n(water) of $0.404\left(\mathrm{D}_{C 4}\right)$ and $0.264\left(\mathrm{E}_{C 4}\right)$, suggest that compartmentation into water- and TBA-rich domains in mixed solvents is responsible for the gel time and the high specific surface area through the modification of the immediate environment of the growing silica polymer. Those ratios $n(T B A) / n($ water $)$ are similar to the ratios in the TBA/water binary mixtures for which structuring has been observed. ${ }^{25,34}$ However, this structuring is different from the effect of structuring due to the templating formed by the aggregation of large amphiphilic molecules. The free energy gain of structure formation in the binary mixtures is very little. Therefore, the introduction of highly hydrophilic silica will influence the morphology of separated TBA-rich and water-rich domains 
instead of forming silica around existing domains. In this case, water-rich domains cover the surface of the growing silica polymer while TBA-rich domains are not in contact with silica. (see Figure 7) The reason is the positive surface charge on the silica surface that exists at the high acid concentrations $(\approx 1 \mathrm{~mol} / \mathrm{L})$ in our experiments ${ }^{64}$. The resulting counter-ion layer of solvated chloride ions supports the hydrophilicity. Thus, the immediate environment of the silica polymer can be considered as a bulk water phase above $x_{\text {water }} \approx 0.5$. As visible by the highest reaction rate observed for $\mathrm{H}_{R E F}$, a solution containing more water has an accelerating effect. The accelerating effect is most pronounced at sample $\mathrm{D}_{C 4}$ because the catalyst concentration in the water-rich domain is the highest. When adding more water, the catalyst in the water-rich domain gets diluted. This modification of the immediate environment appears to have an important effect on the specific surface area. The TBA-rich domains, although strongly fluctuating, are volumes that are excluded for the growing silica polymer. The excluded volumes will lead to pores in the aerogel (see Figure 7) which ultimately lead to unique structural features like observed in the TEM images. The size discrepancy between the solvent structuring in the binary case water/TBA and the observed morphological features is well documented in literature. ${ }^{65,66}$ These excluded volume pores connect the typical microporosity. In the case of unstructured solvents, the microporosity is inaccessible to $\mathrm{N}_{2}$ for measurement. When mesoscale structuring is present, however, microporosity is accessible and leads to a significant increase of specific surface area.

\section{Conclusion}

In this work, the influence of a structured solvent on the kinetics and the resulting morphology of the sol-gel process was investigated. The results are promising for applications in several domains where high specific surface areas are important. ${ }^{6,7,67,68}$ For the investigation, binary mixtures of water/EtOH and water/tert-butanol were chosen as representatives of unstructured (EtOH containing) and structured (tert-butanol containing) solvents. Upon the addition of 
hydrophobic TEOS, enhanced structuring was observed for several samples, which agrees with literature. ${ }^{29}$ After the rapid hydrolysis of TEOS $(<3 \mathrm{~min})$ which was at the origin of the enhanced solvent structuration the solvents are assumed to return to their state in the binary mixtures. Here, the reaction conditions and structuring on the mesoscale determine the size of primary particles. Small silica particles (radii between 0.2 and $0.5 \mathrm{~nm}^{\circ}$ ) then react with each other to form a fractal network. Discontinuity of the reaction rate between the samples was observed in sols containing the structured solvent (tert-butanol) by observation, DLS, FTIR and SAXS. Furthermore, the aerogels obtained by drying with $\mathrm{SC} \mathrm{CO}_{2}$ display noticeable morphological features and specific surface areas when resulting from structured solvents. These criteria lead to the conclusion that the solvent structuring in the immediate environment of the silica particles is responsible for the behavior observed. A hypothesis of the influence of the solvent structuring on the polymerization mechanism is proposed where the silica particles aggregate in a different manner depending on the solvent composition in their close surrounding. In structured solvents water-rich domains cover the silica particles. This leads to reaction conditions that are similar to those in pure water while tert-butanol-rich domains form regions were polymerization does not take place. 


\section{Experimental}

\section{Chemicals}

Ethanol absolute anhydrous (p.a.), acetone (technical grade) and 37 w\% HCL solution (p.a.) were purchased from Carlo Erba Reagents (Val de Reuil, France). Tert-butanol (p.a., $\geq 98$ \%) was purchased from Sigma-Aldrich (Darmstadt, Germany). Tetraethyl orthosilicate (TEOS) ( $\geq$ $98 \%$ ) was purchased from Acros Organics (Geel, Belgium). Chemicals were used without further purification. Aqueous solutions were prepared using Milli-Q water.

\section{Ternary phase diagrams}

Ternary phase diagrams were determined at room temperature using a static and dynamic process as described in literature. ${ }^{29}$ For this purpose, binary mixtures of water/alcohol and alcohol/TEOS were prepared. The third component was added dropwise and the tube was stirred after every addition. The composition at the moment of demixing was noted as the phase boundary. After every addition, the tube was closed and then stirred. To limit the TEOS hydrolysis, ultrapure water was used.

\section{Material preparation}

From the ternary diagrams, several water/alcohol ratios were selected and are referred Acx to Gcx with $X=2$ for ethanol and $X=4$ for tert-butanol (Table 2). For the sample $\mathrm{H}_{R E F}$ no alcohol was added. The materials were prepared using an acid catalyzed sol-gel process. Ethanol or TBA, water and $37 \mathrm{w} \% \mathrm{HCl}$ were mixed in the appropriate ratios indicated in Table 2. After stirring, TEOS was added and the container was closed. . Biphasic samples were vortexed until complete mixing. The longest time required to obtain a monophasic sample was 3 min for $\mathrm{H}_{R E F}$ (Table 2). Afterwards, the sols were kept in an oven at $50^{\circ} \mathrm{C}$ until two days after gelification. Then, the gels were transferred into $100 \mathrm{~mL}$ of ethanol and aged for one day at room temperature. Next, ethanol was decanted from the transparent gels and $100 \mathrm{~mL}$ of acetone were added. After ageing for $24 \mathrm{~h}$, the decanted gels were dried using supercritical (SC) $\mathrm{CO}_{2}$ process. 
This was performed with a SEPAREX supercritical fluid extractor using a $\mathrm{SC} \mathrm{CO}_{2}$ flow of 30 $\mathrm{g} / \mathrm{min}$, at 100 bar and $60^{\circ} \mathrm{C}$ during 3 hours. The obtained white powders were kept in the glove box to prevent degradation. The sample references and compositions are presented in the Table 2 . 
Table 2. References, weight and molar fractions of alcohol and water in the samples containing $7.5 \mathrm{w} \%$ TEOS and $3.6 \mathrm{w} \% \mathrm{HCl}(\mathrm{n}(\mathrm{TEOS}) / \mathrm{n}(\mathrm{HCl})=2.78)$. Their localization in a ternary phase diagram is shown in Figure 1.

\begin{tabular}{|c|c|c|c|c|c|}
\hline \multirow{2}{*}{ Co-solvent } & \multirow{2}{*}{ Reference } & \multicolumn{2}{|c|}{ Weight fractions } & \multicolumn{2}{|c|}{ Molar fractions } \\
\hline & & $w_{\text {water }}$ & $w_{\text {alcohol }}$ & $x_{\text {water }}$ & $x_{\text {alcohol }}$ \\
\hline \multirow{7}{*}{ Ethanol } & $\mathrm{A}_{C 2}$ & 0.1 & 0.79 & 0.24 & 0.71 \\
\hline & $\mathrm{B}_{C 2}$ & 0.15 & 0.74 & 0.32 & 0.63 \\
\hline & $\mathrm{C}_{C 2}$ & 0.24 & 0.65 & 0.47 & 0.49 \\
\hline & $\mathrm{D}_{C 2}$ & 0.33 & 0.56 & 0.58 & 0.38 \\
\hline & $\mathrm{E}_{C 2}$ & 0.43 & 0.46 & 0.68 & 0.29 \\
\hline & $\mathrm{F}_{C 2}$ & 0.61 & 0.28 & 0.82 & 0.15 \\
\hline & $\mathrm{G}_{C 2}$ & 0.8 & 0.09 & 0.93 & 0.04 \\
\hline \multirow{7}{*}{ Tert-butanol } & $\mathrm{A}_{C 4}$ & 0.1 & 0.79 & 0.32 & 0.6 \\
\hline & $\mathrm{B}_{C 4}$ & 0.15 & 0.74 & 0.42 & 0.51 \\
\hline & $\mathrm{C}_{C 4}$ & 0.24 & 0.65 & 0.57 & 0.37 \\
\hline & $\mathrm{D}_{C 4}$ & 0.33 & 0.56 & 0.68 & 0.27 \\
\hline & $\mathrm{E}_{C 4}$ & 0.43 & 0.46 & 0.76 & 0.2 \\
\hline & $\mathrm{F}_{C 4}$ & 0.61 & 0.28 & 0.87 & 0.1 \\
\hline & $\mathrm{G}_{C 4}$ & 0.8 & 0.09 & 0.94 & 0.03 \\
\hline $\begin{array}{c}\text { Ref. without } \\
\text { alcohol }\end{array}$ & $\mathrm{H}_{R E F}$ & 0.89 & 0 & 0.97 & 0 \\
\hline
\end{tabular}


Table 3. Samples used for the SAXS measurements (see Figure 2 b) and d)) All sample contain $7.5 \mathrm{w} \%$ TEOS.

\begin{tabular}{|c|c|c|c|c|c|}
\hline \multirow{2}{*}{ Co-solvent } & \multirow{2}{*}{ Reference } & \multicolumn{2}{|c|}{ Weight fractions } & \multicolumn{2}{|c|}{ Molar fractions } \\
\hline & & $\mathcal{w}_{\text {water }}$ & $w_{\text {alcohol }}$ & $x_{\text {water }}$ & $x_{\text {alcohol }}$ \\
\hline \multirow{5}{*}{$\begin{array}{c}\text { Ethanol } \\
\left(\mathrm{SAXS} \text { at } \mathrm{t}_{0}\right)\end{array}$} & $\mathrm{A}_{C 2} *$ & 0.14 & 0.79 & 0.28 & 0.71 \\
\hline & $\mathrm{B}_{C 2} *$ & 0.19 & 0.74 & 0.36 & 0.63 \\
\hline & $\mathrm{C}_{C 2} *$ & 0.28 & 0.65 & 0.50 & 0.49 \\
\hline & $\mathrm{D}_{C 2} *$ & 0.37 & 0.56 & 0.61 & 0.38 \\
\hline & $\mathrm{E}_{C 2}{ }^{*}$ & 0.46 & 0.46 & 0.70 & 0.29 \\
\hline \multirow{5}{*}{$\begin{array}{l}\text { Tert-butanol } \\
\left(\mathrm{SAXS} \text { at } \mathrm{t}_{0}\right)\end{array}$} & $\mathrm{A}_{C 4} *$ & 0.14 & 0.79 & 0.38 & 0.6 \\
\hline & $\mathrm{B}_{C 4} *$ & 0.19 & 0.74 & 0.47 & 0.51 \\
\hline & $\mathrm{C}_{C 4}{ }^{*}$ & 0.28 & 0.65 & 0.61 & 0.37 \\
\hline & $\mathrm{D}_{C 4} *$ & 0.37 & 0.56 & 0.71 & 0.27 \\
\hline & $\mathrm{E}_{C 4} *$ & 0.46 & 0.46 & 0.79 & 0.2 \\
\hline
\end{tabular}

\section{Characterization techniques}

Gel times were determined by the naked eye and using dynamic light scattering (DLS) at $50^{\circ} \mathrm{C}$ on a Zetasizer Nano ZS (Malvern Panalytical Ltd., United Kingdom) with automatic attenuation in silicone glue sealed $10 \mathrm{~mm}$ polystyrene cuvettes. Acquisition was performed at $173^{\circ}$ for 5 runs of $20 \mathrm{~s}$ each every hour and data were treated using a method adapted from literature. ${ }^{69}$ The time of the last correlation function consisting of a single monomodal exponential function was chosen as gel-time.

Sols were analyzed using Fourier Transformed Infrared spectroscopy (FTIR) with a Perkin Elmer Spectrum 100 spectrometer in Attenuated Total Reflection (ATR) mode equipped with a DTGS/KBR detector. The samples were placed at the surface of the diamond in a PTFE setup to avoid solvent evaporation. The spectra were recorded from 380 to $4000 \mathrm{~cm}^{-1}$ adding 4 
scans with a $4 \mathrm{~cm}^{-1}$ of resolution correcting from the background spectrum for each substrate. Baseline adjustments and peak decomposition were performed using the Origin software.

Small and Wide Angle X-ray Scattering (SWAXS) analysis were carried out in transmission geometry with a Xenocs setup equipped with a Mo anode $(\lambda=0.71 \AA)$ using a MAR345 2D imaging plate detector. Such short wavelength allows to cover a scattering range for standard SWAXS that extends to large wave vectors in order to obtain information at a small scale down to few angstroms typically a wavenumber q ranged from $0.2 \mathrm{~nm}^{-1}$ to $30 \mathrm{~nm}^{-1}$. The collimation was ensured by a Fox2D multilayer mirror and by a set of scatterless slits that delimitated the beam to a square section ( $0.8 \mathrm{~mm}$ side length at the sample position). The distance from the sample to the detector was about $750 \mathrm{~mm}$ and was calibrated using silver behenate powder. Sols were analyzed in glass capillaries of $2 \mathrm{~mm}$ of diameter. Azimuthal averaging of 2D-data recorded by a MAR345 imaging plate detector was performed using the FIT2D software taking into account the electronic background of the detector, the empty cell subtraction and an intensity calibration. The scattered intensity in absolute scale (in $\mathrm{cm}^{-1}$ ) was expressed versus the magnitude of the scattering vector $q=(4 \pi \sin \theta) / \lambda$, where $\theta$ was the scattering angle. Experimental resolution was $\Delta \mathrm{q} / \mathrm{q}=0.02$. Data was fitted using the SASfit software.

Nitrogen adsorption-desorption analyses were carried out using a Micromeritics apparatus (ASAP 2020). Before analysis, all samples were outgassed at $60{ }^{\circ} \mathrm{C}$ during $24 \mathrm{~h}$ under high vacuum $\left(10^{-5} \mathrm{~Pa}\right)$. Specific surface area was obtained using the Brunauer-Emmett-Teller (BET) model.

For transmission electron microscopy (TEM) and high-resolution transmission electron microscopy (HRTEM) measurement, one or more drops of the dilute solution of dried aerogels in ethanol are deposited on the amorphous carbon film. High-resolution transmission electron microscopic measurements were carried out using a JEOL 2200FS microscope that operates at 
$200 \mathrm{kV}$. Transmission electron microscopy observations were then carried out using a FEI Tecnaï G2 TEM microscope equipped with a LaB6 filament that operates at $200 \mathrm{kV}$. 


\section{Associated content}

Supporting Information

The Supporting Information is available and contain the Figures S1-S9 and Table S1 which are related to additional information regarding the phase diagrams, SAXS, FT-IR, TEM analysis and physico-chemical properties of binary systems.

\section{Acknowledgement}

The authors would like to thank Cyrielle Rey and Bruno Corso for the technical support for Nitrogen adsorption-desorption and SAXS analysis, Jingxian Wang and Martiane Cabié for the TEM images and Olivier Diat, Pierre Bauduin, Jeremy Causse, Diane Rebiscoul and Sandrine Dourdain for many helpful and fruitful discussions. 
(1) Riffat, S. B.; Qiu, G. A Review of State-of-the-Art Aerogel Applications in Buildings. Int. J. Low-Carbon Technol. 2013, 8 (1), 1-6. https://doi.org/10.1093/ijlct/cts001.

(2) Plata, D. L.; Briones, Y. J.; Wolfe, R. L.; Carroll, M. K.; Bakrania, S. D.; Mandel, S. G.; Anderson, A. M. Aerogel-Platform Optical Sensors for Oxygen Gas. J. Non. Cryst. Solids 2004, 350, 326-335. https://doi.org/10.1016/j.jnoncrysol.2004.06.046.

(3) Danilyuk, A. .; Kirillov, V. .; Savelieva, M. .; Bobrovnikov, V. .; Buzykaev, A. .; Kravchenko, E. .; Lavrov, A. .; Onuchin, A. . Recent Results on Aerogel Development for Use in Cherenkov Counters. Nucl. Instruments Methods Phys. Res. Sect. A Accel. Spectrometers, Detect. Assoc. Equip. 2002, 494 (1-3), 491-494. https://doi.org/10.1016/S0168-9002(02)01537-1.

(4) Gnade, B.; Cho, C.-C.; Levine, J. D. Low Density, High Porosity Material as Gate Dielectric for Field Emission Device. US5569058A, 1996.

(5) Gurav, J. L.; Jung, I.-K.; Park, H.-H.; Kang, E. S.; Nadargi, D. Y. Silica Aerogel: Synthesis and Applications. J. Nanomater. 2010, 2010, 1-11. https://doi.org/10.1155/2010/409310.

(6) Hu, F.; Wu, S.; Sun, Y. Hollow-Structured Materials for Thermal Insulation. Advanced Materials. John Wiley \& Sons, Ltd October 31, 2018, p 1801001. https://doi.org/10.1002/adma.201801001.

(7) Shi, M.; Tang, C.; Yang, X.; Zhou, J.; Jia, F.; Han, Y.; Li, Z. Superhydrophobic Silica Aerogels Reinforced with Polyacrylonitrile Fibers for Adsorbing Oil from Water and Oil Mixtures. RSC Adv. 2017, 7 (7), 4039-4045. https://doi.org/10.1039/C6RA26831E.

(8) Kim, C.; Baek, S.; Ryu, Y.; Kim, Y.; Shin, D.; Lee, C.-W.; Park, W.; Urbas, A. M.; Kang, G.; Kim, K. Large-Scale Nanoporous Metal-Coated Silica Aerogels for High SERS Effect Improvement. Sci. Rep. 2018, 8 (1), 15144. https://doi.org/10.1038/s41598018-33539-z.

(9) Perdigoto, M. L. N.; Martins, R. C.; Rocha, N.; Quina, M. J.; Gando-Ferreira, L.; Patrício, R.; Durães, L. Application of Hydrophobic Silica Based Aerogels and Xerogels for Removal of Toxic Organic Compounds from Aqueous Solutions. J. Colloid Interface Sci. 2012, 380 (1), 134-140. https://doi.org/10.1016/j.jcis.2012.04.062.

(10) Brinker, C. J.; Scherer, G. W. Sol-Gel Science: The Physics and Chemistry of Sol-Gel Processing; Academic Press: Cambridge, 1990.

(11) Huo, Q.; Margolese, D. I.; Ciesla, U.; Feng, P.; Gier, T. E.; Sieger, P.; Leon, R.; Petroff, P. M.; Schüth, F.; Stucky, G. D. Generalized Synthesis of Periodic Surfactant/Inorganic Composite Materials. Nature 1994, $368 \quad$ (6469), 317-321. https://doi.org/10.1038/368317a0.

(12) Kresge, C. T.; Leonowicz, M. E.; Roth, W. J.; Vartuli, J. C.; Beck, J. S. Ordered Mesoporous Molecular Sieves Synthesized by a Liquid-Crystal Template Mechanism. Nature 1992, 359 (6397), 710-712. https://doi.org/10.1038/359710a0.

(13) Mihaly, M.; Lacatusu, I.; Olteanu, N.-L.; Meghea, A. A Systematic Methodology to Design Silica Templates: Silica Microemulsion Formulation and Nanodroplet Type and Size Estimation. Comptes Rendus Chim. 2014, 17 (4), 342-351. https://doi.org/10.1016/j.crci.2013.09.018. 
(14) Schmidt-Winkel, P.; Glinka, C. J.; Stucky, G. D. Microemulsion Templates for Mesoporous Silica. Langmuir 2000, 16 (2), 356-361. https://doi.org/10.1021/la9906774.

(15) Narayan, R.; Nayak, U.; Raichur, A.; Garg, S. Mesoporous Silica Nanoparticles: A Comprehensive Review on Synthesis and Recent Advances. Pharmaceutics 2018, 10 (3), 118. https://doi.org/10.3390/pharmaceutics 10030118.

(16) Brun, N.; Ungureanu, S.; Deleuze, H.; Backov, R. Hybrid Foams, Colloids and beyond: From Design to Applications. Chem. Soc. Rev. 2011, 40 (2), 771-788. https://doi.org/10.1039/B920518G.

(17) Kunz, W.; Holmberg, K.; Zemb, T. Hydrotropes. Curr. Opin. Colloid Interface Sci. 2016, 22, 99-107. https://doi.org/10.1016/j.cocis.2016.03.005.

(18) Sun, B.; Chai, J.; Chai, Z.; Zhang, X.; Cui, X.; Lu, J. A Surfactant-Free Microemulsion Consisting of Water, Ethanol, and Dichloromethane and Its Template Effect for Silica $\begin{array}{llllll}\text { Synthesis. } J \text {. } & \text { Colloid Interface } & \text { 2018, } & \text { 526, 9-17. }\end{array}$ https://doi.org/10.1016/j.jcis.2018.04.072.

(19) Esquera, J.; Solans, C. No Title Found. Surf. Sci. Ser. 2006, 132, 245-261.

(20) Hayashi, H.; Nishikawa, K.; Iijima, T. Small-Angle X-Ray Scattering Study of Fluctuations in 1-Propanol-Water and 2-Propanol-Water Systems. J. Phys. Chem. 1990, 94 (21), 8334-8338. https://doi.org/10.1021/j100384a062.

(21) Takamuku, T.; Maruyama, H.; Watanabe, K.; Yamaguchi, T. Structure of 1-PropanolWater Mixtures Investigated by Large-Angle X-Ray Scattering Technique. J. Solution Chem. 2004, 33 (6/7), 641-660. https://doi.org/10.1023/B:JOSL.0000043631.21673.8b.

(22) Nishikawa, K.; Iijima, T. Small-Angle X-Ray Scattering Study of Fluctuations in Ethanol and Water Mixtures. J. Phys. Chem. 1993, 97 (41), 10824-10828. https://doi.org/10.1021/j100143a049.

(23) Nishikawa, K.; Hayashi, H.; Iijima, T. Temperature Dependence of the Concentration Fluctuation, the Kirkwood-Buff Parameters, and the Correlation Length of Tert-Butyl Alcohol and Water Mixtures Studied by Small-Angle x-Ray Scattering. J. Phys. Chem. 1989, 93 (17), 6559-6565. https://doi.org/10.1021/j100354a054.

(24) Misawa, M. Mesoscale Structure and Fractal Nature of 1-Propanol Aqueous Solution: A Reverse Monte Carlo Analysis of Small Angle Neutron Scattering Intensity. J. Chem. Phys. 2002, 116 (19), 8463. https://doi.org/10.1063/1.1471903.

(25) Kežić, B.; Perera, A. Aqueous Tert -Butanol Mixtures: A Model for MolecularEmulsions. J. Chem. Phys. 2012, 137 (1), 014501. https://doi.org/10.1063/1.4730524.

(26) Schöttl, S.; Marcus, J.; Diat, O.; Touraud, D.; Kunz, W.; Zemb, T.; Horinek, D. Emergence of Surfactant-Free Micelles from Ternary Solutions. Chem. Sci. 2014, 5 (8), 2949-2954. https://doi.org/10.1039/C4SC00153B.

(27) Klossek, M. L.; Touraud, D.; Kunz, W. Eco-Solvents--Cluster-Formation, Surfactantless Microemulsions and Facilitated Hydrotropy. Phys. Chem. Chem. Phys. 2013, 15 (26), 10971-10977. https://doi.org/10.1039/c3cp50636c.

(28) Sedlák, M.; Rak, D. On the Origin of Mesoscale Structures in Aqueous Solutions of 
Tertiary Butyl Alcohol: The Mystery Resolved. J. Phys. Chem. B 2014, 118 (10), 27262737. https://doi.org/10.1021/jp500953m.

(29) Buchecker, T.; Krickl, S.; Winkler, R.; Grillo, I.; Bauduin, P.; Touraud, D.; Pfitzner, A.; Kunz, W. The Impact of the Structuring of Hydrotropes in Water on the Mesoscale Solubilisation of a Third Hydrophobic Component. Phys. Chem. Chem. Phys. 2017, 19 (3), 1806-1816. https://doi.org/10.1039/C6CP06696H.

(30) Zemb, T. N.; Klossek, M.; Lopian, T.; Marcus, J.; Schöettl, S.; Horinek, D.; Prevost, S. F.; Touraud, D.; Diat, O.; Marčelja, S.; et al. How to Explain Microemulsions Formed by Solvent Mixtures without Conventional Surfactants. Proc. Natl. Acad. Sci. 2016, 113 (16), 4260-4265. https://doi.org/10.1073/pnas.1515708113.

(31) Shimizu, S.; Matubayasi, N. Statistical Thermodynamic Foundation for Mesoscale Aggregation in Ternary Mixtures. Phys. Chem. Chem. Phys. 2018, 20 (20). https://doi.org/10.1039/c8cp01207e.

(32) Shimizu, S.; Matubayasi, N. Unifying Hydrotropy under Gibbs Phase Rule. Phys. Chem. Chem. Phys. 2017, 19 (35), 23597-23605. https://doi.org/10.1039/C7CP02132A.

(33) Kononov, L. O. Chemical Reactivity and Solution Structure: On the Way to a Paradigm Shift? RSC Adv. 2015, 5 (58), 46718-46734. https://doi.org/10.1039/C4RA17257D.

(34) Krickl, S.; Buchecker, T.; Meyer, A. U.; Grillo, I.; Touraud, D.; Bauduin, P.; König, B.; Pfitzner, A.; Kunz, W. A Systematic Study of the Influence of Mesoscale Structuring on the Kinetics of a Chemical Reaction. Phys. Chem. Chem. Phys. 2017, 19 (35), 2377323780. https://doi.org/10.1039/C7CP02134H.

(35) Krickl, S.; Touraud, D.; Bauduin, P.; Zinn, T.; Kunz, W. Enzyme Activity of Horseradish Peroxidase in Surfactant-Free Microemulsions. J. Colloid Interface Sci. 2018, 516, 466475. https://doi.org/10.1016/j.jcis.2018.01.077.

(36) Hou, W.; Xu, J. Surfactant-Free Microemulsions. Curr. Opin. Colloid Interface Sci. 2016, 25, 67-74. https://doi.org/10.1016/j.cocis.2016.06.013.

(37) Yan, X.; Alcouffe, P.; Sudre, G.; David, L.; Bernard, J.; Ganachaud, F. Modular Construction of Single-Component Polymer Nanocapsules through a One-Step Surfactant-Free Microemulsion Templated Synthesis. Chem. Commun. 2017, 53 (8), 1401-1404. https://doi.org/10.1039/C6CC09701D.

(38) Lee, D.-W.; Jin, M.-H.; Park, J.-H.; Lee, Y.-J.; Choi, Y.-C.; Chan Park, J.; Chun, D. H. Alcohol and Water Free Synthesis of Mesoporous Silica Using Deep Eutectic Solvent as a Template and Solvent and Its Application as a Catalyst Support for Formic Acid Dehydrogenation. ACS Sustain. Chem. Eng. 2018, 6 (9), 12241-12250. https://doi.org/10.1021/acssuschemeng.8b02606.

(39) Wang, X.; Xu, J.; Wang, Q.; Xu, A.; Zhai, Y.; Luo, J.; Jiang, Y.; He, N.; Wang, Z. Wet Chemical Synthesis of Silica Nanosheets via Ethyl Acetate-Mediated Hydrolysis of Silica Precursors and Their Applications. Small 2017, 13 (13), 1603369. https://doi.org/10.1002/smll.201603369.

(40) Klossek, M. L.; Touraud, D.; Kunz, W. Eco-Solvents - Cluster-Formation, Surfactantless Microemulsions and Facilitated Hydrotropy. Phys. Chem. Chem. Phys. 2013, 15 (26), 10971. https://doi.org/10.1039/c3cp50636c. 
(41) Shimizu, S.; Matubayasi, N. Hydrotropy: Monomer-Micelle Equilibrium and Minimum Hydrotrope Concentration. J. Phys. Chem. B 2014, 118 (35), 10515-10524. https://doi.org/10.1021/jp505869m.

(42) Clark, G. N. I.; Cappa, C. D.; Smith, J. D.; Saykally, R. J.; Head-Gordon, T. The Structure of Ambient Water. Mol. Phys. 2010, 108 (11), 1415-1433. https://doi.org/10.1080/00268971003762134.

(43) Tomšič, M.; Jamnik, A.; Fritz-Popovski, G.; Glatter, O.; Vlček, L. Structural Properties of Pure Simple Alcohols from Ethanol, Propanol, Butanol, Pentanol, to Hexanol: Comparing Monte Carlo Simulations with Experimental SAXS Data. J. Phys. Chem. B 2007, 111 (7), 1738-1751. https://doi.org/10.1021/jp066139z.

(44) IUPAC Goldbook https://goldbook.iupac.org/html/M/M03853.html (accessed Nov 30, 2018).

(45) Pelster, S. A.; Schrader, W.; Schüth, F. Monitoring Temporal Evolution of Silicate Species during Hydrolysis and Condensation of Silicates Using Mass Spectrometry. $J$. Am. Chem. Soc. 2006, 128 (13), 4310-4317. https://doi.org/10.1021/ja057423r.

(46) Diat, O.; Klossek, M. L.; Touraud, D.; Deme, B.; Grillo, I.; Kunz, W.; Zemb, T. OctanolRich and Water-Rich Domains in Dynamic Equilibrium in the Pre-Ouzo Region of Ternary Systems Containing a Hydrotrope. J. Appl. Crystallogr. 2013, 46 (6), 16651669. https://doi.org/10.1107/S002188981302606X.

(47) Schöttl, S.; Lopian, T.; Prévost, S.; Touraud, D.; Grillo, I.; Diat, O.; Zemb, T.; Horinek, D. Combined Molecular Dynamics (MD) and Small Angle Scattering (SAS) Analysis of Organization on a Nanometer-Scale in Ternary Solvent Solutions Containing a Hydrotrope. J. Colloid Interface Sci. 2019, 540, 623-633. https://doi.org/10.1016/j.jcis.2019.01.037.

(48) Lopian, T.; Schöttl, S.; Prévost, S.; Pellet-Rostaing, S.; Horinek, D.; Kunz, W.; Zemb, T. Morphologies Observed in Ultraflexible Microemulsions with and without the Presence of a Strong Acid. ACS Cent. Sci. 2016, 2 (7), 467-475. https://doi.org/10.1021/acscentsci.6b00116.

(49) Klossek, M. L.; Touraud, D.; Zemb, T.; Kunz, W. Structure and Solubility in SurfactantFree Microemulsions. ChemPhysChem 2012, 13 (18), 4116-4119. https://doi.org/10.1002/cphc.201200667.

(50) Fischer, V.; Marcus, J.; Touraud, D.; Diat, O.; Kunz, W. Toward Surfactant-Free and Water-Free Microemulsions. J. Colloid Interface Sci. 2015, 453, 186-193. https://doi.org/10.1016/j.jcis.2015.04.069.

(51) Meakin, P.; Jullien, R. The Effects of Restructuring on the Geometry of Clusters Formed by Diffusion-limited, Ballistic, and Reaction-limited Cluster-cluster Aggregation. J. Chem. Phys. 1988, 89 (1), 246-250. https://doi.org/10.1063/1.455517.

(52) Ralph K., I. The Chemistry of Silica: Solubility, Polymerization, Colloid and Surface Properties and Biochemistry of Silica; Wiley: New York, 1979.

(53) Aubert, C.; Cannell, D. S. Restructuring of Colloidal Silica Aggregates. Phys. Rev. Lett. 1986, 56 (7), 738-741. https://doi.org/10.1103/PhysRevLett.56.738. 
(54) Kelts, L. W.; Effinger, N. J.; Melpolder, S. M. Sol-Gel Chemistry Studied by $1 \mathrm{H}$ and 29Si Nuclear Magnetic Resonance. J. Non. Cryst. Solids 1986, 83 (3), 353-374. https://doi.org/10.1016/0022-3093(86)90248-6.

(55) Devreux, F.; Boilot, J. P.; Chaput, F.; Lecomte, A. Sol-Gel Condensation of Rapidly Hydrolyzed Silicon Alkoxides: A Joint Si29 NMR and Small-Angle x-Ray Scattering Study. Phys. Rev. A 1990, 41 (12), 6901-6909. https://doi.org/10.1103/PhysRevA.41.6901.

(56) Lim, J.-H.; Ha, S.-W.; Lee, J.-K. Precise Size-Control of Silica Nanoparticles via Alkoxy Exchange Equilibrium of Tetraethyl Orthosilicate (TEOS) in the Mixed Alcohol Solution. Bull. Korean Chem. Soc. 2012, 33 (3), 1067-1070. https://doi.org/10.5012/bkcs.2012.33.3.1067.

(57) Chen, K. C.; Tsuchiya, T.; Mackenzie, J. D. Sol-Gel Processing of Silica. J. Non. Cryst. Solids 1986, 81 (1-2), 227-237. https://doi.org/10.1016/0022-3093(86)90272-3.

(58) He, F.; Zhao, H.; Qu, X.; Zhang, C.; Qiu, W. Modified Aging Process for Silica Aerogel. J. Mater. Process. Technol. 2009, 209 (3), 1621-1626. https://doi.org/10.1016/j.jmatprotec.2008.04.009.

(59) Chou, K.; Lee, B. I. Solvent Effect on Ageing of Silica Gels. J. Mater. Sci. 1994, 29 (13), 3565-3571. https://doi.org/10.1007/BF00352064.

(60) Marlière, C.; Woignier, T.; Dieudonné, P.; Primera, J.; Lamy, M.; Phalippou, J. Two Fractal Structures in Aerogel. J. Non. Cryst. Solids 2001, 285 (1-3), 175-180. https://doi.org/10.1016/S0022-3093(01)00450-1.

(61) Schaefer, D. W.; Keefer, K. D. Fractal Aspects of Ceramic Synthesis. MRS Proc. 1986, 73, 277. https://doi.org/10.1557/PROC-73-277.

(62) Hench, L. L.; West, J. K. The Sol-Gel Process. Chem. Rev. 1990, 90 (1), 33-72. https://doi.org/10.1021/cr00099a003.

(63) Davis, P. J.; Jeffrey Brinker, C.; Smith, D. M.; Assink, R. A. Pore Structure Evolution in Silica Gel during Aging/Drying II. Effect of Pore Fluids. J. Non. Cryst. Solids 1992, 142, 197-207. https://doi.org/10.1016/S0022-3093(05)80026-2.

(64) Duval, Y.; Mielczarski, J. A.; Pokrovsky, O. S.; Mielczarski, E.; Ehrhardt, J. J. Evidence of the Existence of Three Types of Species at the Quartz-Aqueous Solution Interface at PH 0-10: XPS Surface Group Quantification and Surface Complexation Modeling. $J$. Phys. Chem. B 2002, 106 (11), 2937-2945. https://doi.org/10.1021/jp012818s.

(65) Pileni, M.-P. The Role of Soft Colloidal Templates in Controlling the Size and Shape of Inorganic Nanocrystals. Nat. Mater. 2003, 2 (3), 145-150. https://doi.org/10.1038/nmat817.

(66) Richard, B.; Lemyre, J.-L.; Ritcey, A. M. Nanoparticle Size Control in Microemulsion $\begin{array}{lllll}\text { Synthesis. } & \text { Langmuir } & \text { 2017, } & 33 & \text { (19), }\end{array}$ https://doi.org/10.1021/acs.langmuir.7b00773.

(67) Kim, C.; Baek, S.; Ryu, Y.; Kim, Y.; Shin, D.; Lee, C.-W.; Park, W.; Urbas, A. M.; Kang, G.; Kim, K. Large-Scale Nanoporous Metal-Coated Silica Aerogels for High SERS Effect Improvement. Sci. Rep. 2018, 8 (1), 15144. https://doi.org/10.1038/s41598- 
018-33539-z.

(68) Perdigoto, M. L. N.; Martins, R. C.; Rocha, N.; Quina, M. J.; Gando-Ferreira, L.; Patrício, R.; Durães, L. Application of Hydrophobic Silica Based Aerogels and Xerogels for Removal of Toxic Organic Compounds from Aqueous Solutions. J. Colloid Interface Sci. 2012, 380 (1), 134-140. https://doi.org/10.1016/j.jcis.2012.04.062.

(69) Matsunaga, T.; Shibayama, M. Gel Point Determination of Gelatin Hydrogels by Dynamic Light Scattering and Rheological Measurements. Phys. Rev. E 2007, 76 (3), 030401. https://doi.org/10.1103/PhysRevE.76.030401. 